Pacific

Journal of

Mathematics

BOULIGAND DIMENSION AND ALMOST LIPSCHITZ EMBEDDINGS

ERIC OLSON

Volume $202 \quad$ No. 2

February 2002 


\title{
BOULIGAND DIMENSION AND ALMOST LIPSCHITZ EMBEDDINGS
}

\author{
ERIC OLSON
}

\begin{abstract}
In this paper we present some new properties of the metric dimension defined by Bouligand in 1928 and prove the following new projection theorem: $\operatorname{Let} \operatorname{dim}_{b}(\mathcal{A}-\mathcal{A})$ denote the Bouligand dimension of the set $\mathcal{A}-\mathcal{A}$ of differences between elements of $\mathcal{A}$. Given any compact set $\mathcal{A} \subseteq \mathrm{R}^{N}$ such that $\operatorname{dim}_{b}(\mathcal{A}-\mathcal{A})<m$, then almost every orthogonal projection $P$ of $\mathcal{A}$ of rank $m$ is injective on $\mathcal{A}$ and $\left.P\right|_{\mathcal{A}}$ has Lipschitz continuous inverse except for a logarithmic correction term.
\end{abstract}

\section{Introduction.}

What we shall call Bouligand dimension is the dimensional order defined by Bouligand in [4] and further generalized by Assouad much later in [1] and [2]. In this paper we prove several results about Bouligand dimension and its relation to the Mañé type projection theorems of [16], [3] and [10]. The use of Bouligand dimension in studying projections was initiated by Movahedi-Lankarani in [18] where he constructs a set $\mathcal{A}$ with finite fractal dimension for which there are no finite rank projections $P$ with $\left.P\right|_{\mathcal{A}}$ having Lipschitz continuous inverse. Here $\mathcal{A}$ is a subset of a Hilbert space $H$. To do this, he exhibits a set with finite fractal but infinite Bouligand dimension. He then raises the question: What can happen in the case that the Bouligand dimension of $\mathcal{A}$ is finite? This paper will attempt to shed some light on this question.

When $\mathcal{A}$ is an attractor for a partial differential equation, knowing whether $\mathcal{A}$ may be embedded into a finite dimensional space is of theoretical and computational interest. Work along these lines include Eden, Foias, Nicolaenko and Temam [8] on exponential attractors, Sauer, Yorke and Casdagli [23] on delay-coordinate maps, and Robinson [21], [20] on approximate attractors. Ideally, given an infinite dimensional dynamical system, we would like to construct a finite dimensional dynamical system that has the same long-term behavior. This is important, in particular, because any numerical simulation of the dynamics on a computer is by necessity finite dimensional. Since the long-term behavior of a dynamical system is largely governed by its global attractor, this question may be rephrased as whether it is possible to 
construct a finite dimensional dynamical system with the same global attractor as the original dynamical system. To construct such a system requires embedding the attractor of the original infinite dimensional dynamical system into a finite dimensional space. This embedding should posses certain continuity properties so as to preserve the original dynamics.

Inertial manifolds, as discussed by Constantin in [5] and Constantin, Foias, Nicolaenko and Temam in [6] and [7], provide a bi-Lipschitz embedding of the global attractor into a finite dimensional space. As the Bouligand dimension of an attractor is preserved under bi-Lipschitz mappings, the following holds:

Fact 1.1. The Bouligand dimension of the global attractor of any dynamical system possessing an inertial manifold must be finite.

Thus, there exist a number of global attractors with finite Bouligand dimension. For example, the Kuramoto-Sivashinsky equation, the Kolmogorov-Sivashinsky-Spiegel equation and the Ginzburg-Landau equation in one space dimension [7], reaction diffusion equations in higher space dimensions [15], and nonlinear viscoelasticity equations [19] have inertial manifolds and therefore global attractors with finite Bouligand dimension. It not known if there exists an inertial manifold for the Navier-Stokes equations in two space dimensions. In light of the main result in this paper, computing the Bouligand dimension of the global attractor directly and more specifically the Bouligand dimension of the set $\mathcal{A}-\mathcal{A}$ of differences between elements of $\mathcal{A}$ is of great interest. Our main result is:

Theorem 1.2. Given $\mathcal{A} \subseteq \mathbf{R}^{N}$ such that $\operatorname{dim}_{b}(\mathcal{A}-\mathcal{A})<m$, then almost every orthogonal projection $P$ of $\mathcal{A}$ of rank $m$ is injective on $\mathcal{A}$ and $\left.P\right|_{\mathcal{A}}$ has Lipschitz continuous inverse except for a logarithmic correction term.

This shows that almost every orthogonal projection of $\mathcal{A}$ has the same Lipschitz properties that the embeddings of an inertial manifold have except for a logarithmic correction. Here, almost every should be understood in terms of the Haar measure invariant with respect to orthogonal transformations on the space of all orthogonal projections of rank $m$ in $\mathbf{R}^{n}$. This provides a partial converse to Fact 1.1. Note that $n$ may be chosen arbitrarily large. Thus, Theorem 1.2 embeds a fractal subset $\mathcal{A}$ of a large finite dimensional space into a smaller finite dimensional space whose dimension is controlled explicitly by the Bouligand dimension of $\mathcal{A}-\mathcal{A}$. Our theorem is related to a result stated by Mañé in [16] which states under the assumption of finite Hausdorff dimension that the injective projections form residual set.

Definition 1.3. The Hausdorff dimension of the set $\mathcal{A}$ is defined by

$$
\operatorname{dim}_{h}(\mathcal{A})=\inf \left\{d: \mathcal{H}^{d}(\mathcal{A})=0\right\}=\sup \left\{d: \mathcal{H}^{d}(\mathcal{A})=\infty\right\}
$$


where $\mathcal{H}^{d}$ is the $d$-dimensional Hausdorff measure

$$
\mathcal{H}^{d}(\mathcal{A})=\lim _{\delta \rightarrow 0} \inf \left\{\sum_{i=1}^{\infty}\left|U_{i}\right|^{d}:\left\{U_{i}\right\} \text { is a } \delta \text {-cover of } \mathcal{A}\right\} .
$$

Here $\left|U_{i}\right|=\sup \left\{|x-y|: x, y \in U_{i}\right\}$ is the diameter of the set $U_{i}$ and a $\delta$-cover is a cover $\left\{U_{i}\right\}$ such that $\left|U_{i}\right| \leq \delta$ for all $i$.

Definition 1.4. A set is called residual if its complement is a set of first category; a set of first category is a countable union of nowhere dense sets; and a nowhere dense set is a set whose closure has no interior points.

Theorem 1.5 (Mañé). If $E$ is a Banach space and $\mathcal{A} \subset E$ is a countable union of compact subsets with $\operatorname{dim}_{h}(\mathcal{A})<\infty$ then for every subspace $\mathcal{B}$ of $E$ with $\operatorname{dim}_{h}(\mathcal{A}-\mathcal{A})+1<\operatorname{dim}(\mathcal{B})<\infty$ the set of projections $P: \mathcal{A} \rightarrow \mathcal{B}$ such that $\left.P\right|_{\mathcal{A}}$ is injective is a residual subset of the space of projections of $\mathcal{A}$ onto $\mathcal{B}$ endowed with the norm topology.

Hölder continuity for the inverse in Mañé's projection theorem has been proved under the hypothesis of finite fractal dimension by Ben-Artzi, Eden, Foias, and Nicolaenko in [3], and extended by Foias and Olson in [10].

Definition 1.6. Let $\mathcal{N}_{\mathcal{A}}(\rho)$ be the minimum number of $\rho$-radius balls required to cover all of $\mathcal{A}$. Then, the fractal dimension

$$
\operatorname{dim}_{f}(\mathcal{A})=\limsup _{\rho \rightarrow 0} \frac{\log \mathcal{N}_{\mathcal{A}}(\rho)}{\log \rho} .
$$

Theorem 1.7 (Foias and Olson). Let $H$ be a real Hilbert space and $\mathcal{A} \subseteq H$ be such that $2 \operatorname{dim}_{f}(\mathcal{A})<m$. Then for any orthogonal projection $P$ of rank $m$ and $\delta>0$ there is an orthogonal projection $\widetilde{P}$ such that $\|\widetilde{P}-P\|<\delta$ and $\left.\widetilde{P}\right|_{\mathcal{A}}$ has Hölder inverse.

Hunt and Kaloshin in $[\mathbf{1 3}]$ have recently shown that such projections $\widetilde{P}$ are in fact prevalent according to the sense of prevalence given by Hunt, Sauer and Yorke in [14]. Prevalence extends the notion of Lebesgue almost every from Euclidean spaces to infinite-dimensional spaces. In particular:

Definition 1.8. A Borel subset $S \subseteq B$ is prevalent if there exists a compactly supported probability measure $\mu$ such that $\mu(S+x)=1$ for all $x \in B$. A set, in general, is prevalent if it contains a prevalent Borel set.

Note that Theorem 1.7 is stated with a hypothesis only on the fractal dimension of $\mathcal{A}$. This simplification may be made because the fractal dimension of $\mathcal{A}-\mathcal{A}$ is controlled by the inequality $\operatorname{dim}_{f}(\mathcal{A}-\mathcal{A})<2 \operatorname{dim}_{f}(\mathcal{A})$. Such inequalities are not true for the Bouligand and Hausdorff dimensions. Therefore, Theorems 1.2 and 1.5 are stated under hypotheses about the set $\mathcal{A}-\mathcal{A}$ directly. In this paper we give an example for Bouligand dimension 
for which $\operatorname{dim}_{b}(\mathcal{A})=0$ and $\operatorname{dim}_{b}(\mathcal{A}-\mathcal{A})=\infty$ to demonstrate this dramatic difference.

We also show the Bouligand dimension of a set may increase under an orthogonal projection. Note that for the fractal and Hausdorff dimensions, the dimension of the projected image is always less than or equal the dimension of the original set. Thus, Bouligand dimension gives us further insight over what can happen to the geometry of a set under orthogonal projection. On the other hand, there may be bad projections that make a global attractor appear more complicated than it really is.

This paper is organized as follows: First, we define Bouligand dimension and give an alternate characterization of it. In Section 3 we state some properties and show that Bouligand dimension agrees with other notions of dimension for self-similar fractals. In Section 4 we prove Theorem 4.7 and in Section 5 we prove Theorem 1.2.

\section{The Bouligand dimension.}

In this section we define the fractal and Bouligand dimensions. Fractal dimension is sometimes also called the box-counting dimension, the capacity, or the Minkowski dimension. We have already defined this dimension in the introduction via Definition 1.6. An equivalent characterization of fractal dimension in terms of an infimum follows easily from the definitions of infimum and limit superior.

Fact 2.1. The fractal $\operatorname{dimension} \operatorname{dim}_{f}(\mathcal{A})$ is the infimum over all $d$ for which there exists $K$ such that

$$
\mathcal{N}_{\mathcal{A}}(\rho) \leq K(1 / \rho)^{d} \quad \text { for } 0<\rho<1 .
$$

We now define Bouligand dimension. As already mentioned this dimension is the generalization of the dimensional order of Bouligand [4] discussed by Assouad in [1] and [2] and by Movahedi-Lankarani in [18].

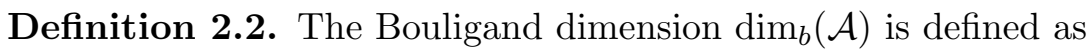

$$
\operatorname{dim}_{b}(\mathcal{A})=\lim _{\epsilon \rightarrow 0} \lim _{t \rightarrow \infty} \Delta_{\epsilon, t}(\mathcal{A})
$$

where

$$
\Delta_{\epsilon, t}(\mathcal{A})=\sup \left\{\frac{\log \mathcal{N}(r, \rho)}{\log (r / \rho)}: 0<\rho<r<\epsilon \text { and } r>t \rho\right\}
$$

and $\mathcal{N}(r, \rho)$ is the number of $\rho$-balls required to cover any $r$-ball in $\mathcal{A}$.

The Bouligand dimension may be characterized as an infimum in a way similar to that of the fractal dimension. This is essentially the definition given by Assouad in [1]. This characterization highlights a scaling condition that will be important later. In particular, given $0<\lambda \leq 1$ the Bouligand dimension requires the number of $\rho$ balls needed to cover an $r$ ball portion 
of the set should be essentially the same as the number of $\lambda \rho$ balls needed to cover a $\lambda r$ ball portion.

Theorem 2.3. The Bouligand dimension $\operatorname{dim}_{b}(\mathcal{A})$ is the infimum over all $d$ for which there exists $K$ such that

$$
\mathcal{N}_{\mathcal{A}}(r, \rho) \leq K(r / \rho)^{d} \quad \text { for } 0<\rho<r<1 .
$$

First note that the exact form of the upper bound for $r$ in condition (2.2) is not critical. Equivalently we may require for some $\epsilon>0$ that there exists $K_{\epsilon}$ such that

$$
\mathcal{N}_{\mathcal{A}}(r, \rho) \leq K_{\epsilon}(r / \rho)^{d} \quad \text { for } 0<\rho<r<\epsilon .
$$

It is obvious that if $\epsilon<1$, then condition (2.2) implies (2.3) with $K_{\epsilon}=K$. Conversely suppose (2.3) holds for some $0<\epsilon<1$. Since $\mathcal{A}$ is compact, then a finite number $N$ of $\epsilon / 4$ balls will cover it. Let $r$ and $\rho$ be such that $0<\rho<r<1$. Consider the following cases:

Case $\rho \geq \epsilon / 2$. Then

$$
\mathcal{N}_{\mathcal{A}}(r, \rho) \leq \mathcal{N}_{\mathcal{A}}(r, \epsilon / 2) \leq \mathcal{N}_{\mathcal{A}}(\epsilon / 4) \leq N .
$$

Case $\rho<\epsilon / 2 \leq r$. Then

$$
\mathcal{N}_{\mathcal{A}}(r, \rho) \leq \mathcal{N}_{\mathcal{A}}(r, \epsilon / 2) \mathcal{N}_{\mathcal{A}}(\epsilon / 2, \rho) \leq N K_{\epsilon}\left(\frac{\epsilon / 2}{\rho}\right)^{d} \leq N K_{\epsilon}\left(\frac{r}{\rho}\right)^{d} .
$$

Thus taking $K=N K_{\epsilon}$ we obtain that

$$
\mathcal{N}_{\mathcal{A}}(r, \rho) \leq K\left(\frac{r}{\rho}\right)^{d} \quad \text { for } 0<\rho<r<1 .
$$

In the situation where $\epsilon>1$ the argument is similar. Moreover, if there exists one $\epsilon$ and $K_{\epsilon}$ for which condition (2.3) holds, then for each $\epsilon$ there is a $K_{\epsilon}$ such that it holds.

Proof of Theorem 2.3. Suppose there is $d$ and $K$ such that (2.2) holds. Then

$$
\frac{\log \mathcal{N}_{\mathcal{A}}(r, \rho)}{\log (r / \rho)} \leq d+\frac{\log K}{\log (r / \rho)} \quad \text { for } 0<\rho<r<1 .
$$

It follows that

$$
\Delta_{1, t}(\mathcal{A}) \leq d+\frac{\log K}{\log (t)}
$$

and therefore

$$
\operatorname{dim}_{b}(\mathcal{A})=\lim _{\epsilon \rightarrow 0} \lim _{t \rightarrow \infty} \Delta_{1, t}(\mathcal{A}) \leq d .
$$


Conversely, suppose $d$ is chosen so that $\operatorname{dim}_{b}(\mathcal{A})<d$. Then by definition there are values of $\epsilon$ and $t$ such that $0<\epsilon<1<t$ and $\Delta_{\epsilon, t}(\mathcal{A})<d$. It follows that

$$
\frac{\log \mathcal{N}_{\mathcal{A}}(r, \rho)}{\log (r / \rho)}<d \quad \text { for } 0<t \rho<r<\epsilon
$$

and therefore

$$
\mathcal{N}_{\mathcal{A}}(r, \rho)<(r / \rho)^{d} \quad \text { for } 0<t \rho<r<\epsilon .
$$

Now, suppose $0<\rho<r<\epsilon$. Then $0<t(\rho / t)<r<\epsilon$ and

$$
\mathcal{N}_{\mathcal{A}}(r, \rho) \leq \mathcal{N}_{\mathcal{A}}(r, \rho / t) \leq\left(\frac{r}{\rho / t}\right)^{d}=K\left(\frac{r}{\rho}\right)^{d} \quad \text { for } 0<\rho<r<\epsilon
$$

where $K=t^{d}$. It follows that $\operatorname{dim}_{b}(\mathcal{A})$ is the infimum over all $d$ for which (2.2) holds.

It is often of interest to know whether a set has finite Bouligand dimension or not. The following lemma provides a simple test for determining this.

Lemma 2.4. If there exists $K$ such that $\mathcal{N}_{\mathcal{A}}(r, r / 2)<K$ holds for all $r<1$ then $\mathcal{A}$ has finite Bouligand dimension. Moreover, $\operatorname{dim}_{b}(\mathcal{A}) \leq \log _{2} K$.

Proof. Given $r$ and $\rho$ such that $0<\rho<r<1$ choose $n$ so that $r / 2^{n} \leq \rho<$ $r / 2^{n-1}$. Then

$$
\mathcal{N}_{\mathcal{A}}(r, \rho) \leq \mathcal{N}_{\mathcal{A}}(r, r / 2) \mathcal{N}_{\mathcal{A}}\left(r / 2, r / 2^{2}\right) \cdots \mathcal{N}_{\mathcal{A}}\left(r / 2^{n-1}, r / 2^{n}\right) \leq K^{n}
$$

and since $n-1 \leq \log _{2}(r / \rho)$ it follows that

$$
\mathcal{N}_{\mathcal{A}}(r, \rho) \leq K\left(K^{n-1}\right) \leq K(r / \rho)^{\log _{2} K} .
$$

Therefore $\operatorname{dim}_{b}(\mathcal{A}) \leq \log _{2} K<\infty$.

\section{Properties of the Bouligand dimension.}

The Bouligand dimension satisfies many of the usual properties that a reasonable dimension should satisfy. In this section we state and prove a few of these properties. In particular, we prove the Bouligand dimension is well behaved with respect to Cartesian products and that the Bouligand dimension agrees with the similarity dimension for self-similar fractals.

First, we will state for reference as Theorem 3.1 a few properties of the Bouligand dimension found in Movahedi-Lankarani [18] and Assouad [1].

Theorem 3.1. The Bouligand dimension has the following properties:

(i) If $\mathcal{A} \subseteq \mathcal{B}$ then $\operatorname{dim}_{b}(\mathcal{A}) \leq \operatorname{dim}_{b}(\mathcal{B})$.

(ii) If $\mathcal{A}$ is an open subset of $\mathbf{R}^{N}$ then $\operatorname{dim}_{b}(\mathcal{A})=N$.

(iii) If $\mathcal{A}$ and $\mathcal{B}$ are bi-Lipschitz isomorphic, then $\operatorname{dim}_{b}(\mathcal{A})=\operatorname{dim}_{b}(\mathcal{B})$. 
We now discuss how the Bouligand dimension behaves with respect to Cartesian products. We would like

$$
\operatorname{dim}_{b}(\mathcal{A} \times \mathcal{B})=\operatorname{dim}_{b}(\mathcal{A})+\operatorname{dim}_{b}(\mathcal{B}) .
$$

However, as with any metric dimension, the Bouligand dimension depends on what metric is used. Therefore, care must be taken in choosing a metric. Let $\mathcal{A}$ and $\mathcal{B}$ be compact metric spaces with metrics $d_{\mathcal{A}}$ and $d_{\mathcal{B}}$. Define the metric spaces $X_{p}=\mathcal{A} \times \mathcal{B}$ for $1 \leq p \leq \infty$ by the metrics $d_{p}$ given by

$$
d_{p}\left(\left(a_{1}, b_{1}\right),\left(a_{2}, b_{2}\right)\right)= \begin{cases}\left(d_{\mathcal{A}}\left(a_{1}, a_{2}\right)^{p}+d_{\mathcal{B}}\left(b_{1}, b_{2}\right)^{p}\right)^{1 / p} & \text { for } 1 \leq p<\infty \\ \max \left(d_{\mathcal{A}}\left(a_{1}, a_{2}\right), d_{\mathcal{B}}\left(b_{1}, b_{2}\right)\right) & \text { for } p=\infty\end{cases}
$$

Theorem 3.2. $\operatorname{dim}_{b}\left(X_{p}\right)=\operatorname{dim}_{b}(\mathcal{A})+\operatorname{dim}_{b}(\mathcal{B})$.

Proof. Since $d_{\infty}$ is Lipschitz equivalent to $d_{p}$ for $1 \leq p<\infty$, it follows from property (iii) of Theorem 3.1 that

$$
\operatorname{dim}_{b}\left(X_{\infty}\right)=\operatorname{dim}_{b}\left(X_{p}\right) \quad \text { for } \quad 1 \leq p<\infty .
$$

Hence, we may prove the theorem for $X=X_{\infty}$ without loss of generality.

For every $\alpha$ and $\beta$ such that $\operatorname{dim}_{b}(\mathcal{A})<\alpha$ and $\operatorname{dim}_{b}(\mathcal{B})<\beta$ there exists $K_{\mathcal{A}}$ and $K_{\mathcal{B}}$ such that

$$
\mathcal{N}_{\mathcal{A}}(r, \rho)<K_{\mathcal{A}}\left(\frac{r}{\rho}\right)^{\alpha} \quad \text { and } \quad \mathcal{N}_{\mathcal{B}}(r, \rho)<K_{\mathcal{B}}\left(\frac{r}{\rho}\right)^{\beta}
$$

for $0<\rho<r<1$. Let $B$ be a ball of radius $r$ in $X$. Then by definition, $B=U \times V$ where $U$ and $V$ are balls of radius $r$. Cover $U$ by balls $U_{i}$ of radius $\rho$ in $\mathcal{A}$ and $V$ by balls $V_{j}$ of radius $\rho$ in $\mathcal{B}$. Since $U_{i} \times V_{j}$ form a cover of $B$, it follows that

$$
\mathcal{N}_{X}(r, \rho) \leq \mathcal{N}_{\mathcal{A}}(r, \rho) \mathcal{N}_{\mathcal{B}}(r, \rho) \leq K_{\mathcal{A}} K_{\mathcal{B}}\left(\frac{r}{\rho}\right)^{\alpha+\beta} .
$$

Hence $\operatorname{dim}_{b}(X) \leq \alpha+\beta$ and so $\operatorname{dim}_{b}(X) \leq \operatorname{dim}_{b}(\mathcal{A})+\operatorname{dim}_{b}(\mathcal{B})$.

Now, let $n$ be the maximum number of disjoint $\rho / 2$-radius balls with centers $u_{i}$ in $U$. Let $U_{i}$ be balls of radius $\rho$ with the same centers. Thus the points $u_{i}$ are at least a distance $\rho / 2$ apart from each other and $U_{i}$ covers $U$. Do the same for $V$ to obtain $m$ balls $V_{j}$ of radius $\rho$ whose centers $v_{j}$ are at least a distance $\rho / 2$ apart from each other which cover $V$. Let $z_{k} \in B$ be an enumeration of the points $\left(u_{i}, v_{j}\right)$ and define $Z=\left\{z_{k}: k=1 \ldots n m\right\}$. It follows that

$$
\mathcal{N}_{X}(r, \rho / 4) \geq \mathcal{N}_{Z}(\rho / 2) \geq n m \geq \mathcal{N}_{U}(\rho) \mathcal{N}_{V}(\rho) .
$$

Choose $U$ and $V$ such that $\mathcal{N}_{U}(\rho)$ and $\mathcal{N}_{V}(\rho)$ attain their maximum values. Given $d$ such that $\operatorname{dim}_{b}(X)<d$, there is $K$ such that

$$
\mathcal{N}_{X}(r, \rho) \leq K\left(\frac{r}{\rho}\right)^{d} \quad \text { for } 0<\rho<r<1 .
$$


Thus

$$
\mathcal{N}_{\mathcal{A}}(r, \rho) \mathcal{N}_{\mathcal{B}}(r, \rho) \leq \mathcal{N}_{X}(r, \rho / 4) \leq 4^{d} K\left(\frac{r}{\rho}\right)^{d},
$$

and therefore by Theorem $2.3 \operatorname{dim}_{b}(\mathcal{A})+\operatorname{dim}_{b}(\mathcal{B})=\operatorname{dim}_{b}(X)$.

It is interesting to note that the Hausdorff dimension does not behave well under Cartesian products. For example, Falconer exhibits sets $\mathcal{A}$ and $\mathcal{B}$ in $[9] \operatorname{such}$ that $\operatorname{dim}_{h}(\mathcal{A})+\operatorname{dim}_{h}(\mathcal{B})<\operatorname{dim}_{h}(\mathcal{A} \times \mathcal{B})$.

We end this section with a calculation of the Bouligand dimension for a self-similar fractal. In particular, we show the Bouligand dimension agrees with the similarity dimension for such sets, and therefore the fractal and Hausdorff dimensions as well. First note that the Hausdorff dimension is bounded by the fractal dimension which is in turn bounded by the Bouligand dimension. Thus given a compact metric space $\mathcal{A}$ we have that

$$
\operatorname{dim}_{h}(\mathcal{A}) \leq \operatorname{dim}_{f}(\mathcal{A}) \leq \operatorname{dim}_{b}(\mathcal{A}) .
$$

Definition 3.3. A transformation $f: \mathbf{R}^{n} \rightarrow R^{n}$ is said to be a similarity if there is a constant $c$ such that $|f(x)-f(y)|=c|x-y|$ for all $x, y$ in $R^{n}$.

Let $f_{i}: \mathbf{R}^{N} \rightarrow \mathbf{R}^{N}$, where $i=1, \ldots, L$ be similarities with ratios $c_{i} \in$ $(0,1)$ and define $F(M)=\cup f_{i}(M)$ for $M \subseteq \mathbf{R}^{N}$. Let $\mathcal{A}$ be the compact invariant set such that $F(\mathcal{A})=\mathcal{A}$.

Definition 3.4. The similarity dimension of $\mathcal{A}$ is defined as $\operatorname{dim}_{s}(\mathcal{A})=s$ where $\Sigma c_{i}^{s}=1$.

Theorem 3.5. Let $\mathcal{A}$ be defined as above. Then $\operatorname{dim}_{b}(\mathcal{A})=\operatorname{dim}_{s}(\mathcal{A})$.

Proof. Since $\mathcal{A}$ is compact there is $\epsilon$ such that $0<\epsilon<1$ and

$$
\operatorname{dist}\left(f_{i}(\mathcal{A}), f_{j}(\mathcal{A})\right)>\epsilon \quad \text { for } \quad i \neq j .
$$

Let $c=\min \left\{c_{i}: i=1, \ldots, L\right\}$. Let $R>\epsilon / c$ be chosen so large that $\mathcal{A}$ fits inside an $R$ ball. Let $r$ and $\rho$ be chosen so that $0<\rho<r<\epsilon / 2$. Let $I$ be the set of finite sequences $\left(i_{1}, \ldots, i_{n}\right)$ such that

$$
c_{i_{1}} \cdots c_{i_{n}} \epsilon \leq 2 r<c_{i_{1}} \cdots c_{i_{n-1}} \epsilon .
$$

Since $2 r<\epsilon$, then $I$ is nonempty. Furthermore, $\mathcal{A}=\cup\left\{f_{i_{1}} \circ \cdots \circ f_{i_{n}}(\mathcal{A})\right.$ : $\left.\left(i_{1}, \ldots, i_{n}\right) \in I\right\}$. Let $\left(i_{1}, \ldots, i_{n}\right)$ and $\left(j_{1}, \ldots, j_{m}\right)$ be distinct elements of $I$. Suppose for definiteness that $n \leq m$. Consider the sets $f_{i_{1}} \circ \cdots \circ f_{i_{n}}(\mathcal{A})$ and $f_{j_{1}} \circ \cdots \circ f_{j_{m}}(\mathcal{A})$. Let $k$ be the largest index such that $i_{l}=j_{l}$ for all $l \leq k$. Obviously $k<n$ for otherwise $k=n$ would imply $n<m$ leading to the contradiction

$$
c_{i_{1}} \cdots c_{i_{n}} \epsilon \leq 2 r<c_{j_{1}} \cdots c_{j_{m-1}} \epsilon \leq c_{j_{1}} \cdots c_{j_{n}} \epsilon=c_{i_{1}} \cdots c_{i_{n}} \epsilon .
$$


Define $\mathcal{F}=f_{i_{k}+1} \circ \cdots \circ f_{i_{n}}(\mathcal{A})$ and $\mathcal{G}=f_{j_{k}+1} \circ \cdots \circ f_{j_{m}}(\mathcal{A})$. Then $i_{k+1} \neq j_{k+1}$ implies $\operatorname{dist}(\mathcal{F}, \mathcal{G})>\epsilon$. It follows that

$$
\operatorname{dist}\left(f_{i_{1}} \circ \cdots \circ f_{i_{n}}(\mathcal{A}), f_{j_{1}} \circ \cdots \circ f_{j_{m}}(\mathcal{A})\right)>c_{i_{1}} \cdots c_{i_{k}} \epsilon \geq c_{i_{1}} \cdots c_{i_{n-1}} \epsilon>2 r .
$$

Now let $B$ be a ball of radius $r$. It follows that $B \cap f_{i_{1}} \circ \cdots \circ f_{i_{n}}(\mathcal{A}) \neq \emptyset$ for at most one $\left(i_{1}, \ldots, i_{n}\right) \in I$. Let $\left(i_{1}, \ldots, i_{n}\right)$ be that one and fix it.

Hence $B \cap \mathcal{A}=B \cap f_{i_{1}} \circ \cdots \circ f_{i_{n}}(\mathcal{A})$. Define $\gamma=c_{i_{1}} \cdots c_{i_{n}}$ and let $J$ be the set of $\left(j_{1}, \ldots, j_{m}\right)$ such that

$$
R \gamma c_{j_{1}} \cdots c_{j_{m}} \leq \rho<R \gamma c_{j_{1}} \cdots c_{j_{m-1}} .
$$

Since $\rho<r \leq \gamma \epsilon / c \leq R \gamma$, then $J$ is nonempty. Since

$$
\mathcal{A}=\cup\left\{f_{j_{1}} \circ \cdots \circ f_{j_{m}}(\mathcal{A}):\left(j_{1}, \ldots, j_{n}\right) \in J\right\}
$$

it follows that

$$
B \cap \mathcal{A} \subseteq \cup\left\{f_{i_{1}} \circ \cdots \circ f_{i_{n}} \circ f_{j_{1}} \circ \cdots \circ f_{j_{m}}(\mathcal{A}):\left(j_{1}, \ldots, j_{m}\right) \in J\right\} .
$$

Furthermore, since $R \gamma c_{j_{1}} \cdots c_{j_{m}} \leq \rho$, it follows that

$$
f_{i_{1}} \circ \cdots \circ f_{i_{n}} \circ f_{j_{1}} \circ \cdots \circ f_{j_{m}}(\mathcal{A}) \subseteq B_{\rho}\left(f_{i_{1}} \circ \cdots \circ f_{i_{n}} \circ f_{j_{1}} \circ \cdots \circ f_{j_{m}}(\alpha)\right) .
$$

We now estimate the number of elements in $J$. Since $\Sigma c_{i}^{s}=1$, it follows by induction that $\sum_{J}\left(c_{j_{1}} \cdots c_{j_{m}}\right)^{s}=1$. Therefore $\sum_{J}(c \rho /(R \gamma))^{s} \leq 1$ and so $J$ has no more than $(R \gamma /(c \rho))^{s}$ elements. Hence, $\mathcal{N}_{B \cap \mathcal{A}}(\rho) \leq(R \gamma /(c \rho))^{s}$. Since $\gamma=c_{i_{1}} \cdots c_{i_{n}} \leq 2 r / \epsilon$, it follows that $\mathcal{N}_{\mathcal{A}}(r, \rho) \leq K(r / \rho)^{s}$ where $K=$ $(2 R /(c \epsilon))^{s}$. As this holds for all $0<\rho<r<\epsilon / 2$, it further follows that $\operatorname{dim}_{b}(\mathcal{A}) \leq s$. By $[\mathbf{9}]$, Theorem 9.3, the Hausdorff $\operatorname{dimension}_{\operatorname{dim}}(\mathcal{A})=s$; therefore, in light of $(3.1)$ we obtain that $\operatorname{dim}_{b}(\mathcal{A})=s$.

Thus, for self-similar sets, the Bouligand dimension agrees with the similarity, fractal, and Hausdorff dimensions. In the case that $c_{i}=c$ for all $i$, the similarity dimension has an easy to calculate form and we obtain the following corollary:

Corollary 3.6. Let $f_{i}: \mathbf{R}^{N} \rightarrow \mathbf{R}^{N}$ where $i=1, \ldots, L$ be similarities with ratio $c \in(0,1)$ and define $F(M)=\cup f_{i}(M)$ for $M \subseteq \mathbf{R}^{N}$. Take $\mathcal{A}$ to be the compact invariant set such that $F(\mathcal{A})=\mathcal{A}$. If the images under the $f_{i}$ are disjoint then $\operatorname{dim}_{b}(\mathcal{A})=-\log (L) / \log (c)$.

\section{Dimension increasing projections.}

The results in this section are motivated by examples involving orthogonal sequences in a Hilbert space. The fractal dimension of such sequences has been already studied by Ben-Artzi, Eden, Foias and Nicolaenko in [3] and by Ladislav Mišík Jr. and Tibor Žáčik in [17]. In some sense, an orthogonal sequence is the farthest thing possible from the regular self-similar fractals discussed in the previous section. Not surprisingly, it is in the treatment of 
these sets that the Bouligand dimension differs most dramatically from the fractal dimension. In particular, we exhibit a set $\mathcal{A} \operatorname{such}$ that $\operatorname{dim}_{b}(\mathcal{A})=0$ and $\operatorname{dim}_{b}(\mathcal{A}-\mathcal{A})=\infty$. This section closes with a proof of Theorem 4.7 concerning the existence orthogonal projections that increase the Bouligand dimension.

In Theorem 2.3 it was shown that the Bouligand dimension requires that the number of $\rho$ balls needed to cover an $r$ ball should be essentially the same as than the number of $\lambda \rho$ balls needed to cover a $\lambda r$ ball. The homogeneity of this scaling makes the Bouligand dimension sensitive to inhomogeneities in the set $\mathcal{A}$. We will now consider a particularly inhomogeneous set: The closure of an orthogonal sequence converging to zero in a Hilbert space. Let $H$ be a Hilbert space and $e_{i}$ an orthonormal sequence.

Lemma 4.1. Let $\mathcal{A}=\{0\} \cup\left\{e_{n} / n^{\alpha}: n \in \mathbf{N}\right\}$. Then $\operatorname{dim}_{f}(\mathcal{A})=1 / \alpha$ and $\operatorname{dim}_{b}(\mathcal{A})=\infty$.

Proof. The first fact appears in [3]. For the second, consider the ball of radius $r=1 / m^{\alpha}$ centered at the origin

$$
B=\{a \in \mathcal{A}:\|a\|<r\}=\{0\} \cup\left\{e_{n} / n^{\alpha}: n>m\right\} .
$$

Cover $B$ by $r / 2$ balls. Each point a distance more that $r / 2$ from the origin will require a separate ball. Since

$$
1 / n^{\alpha}>r / 2 \quad \text { implies } \quad n<(2 / r)^{1 / \alpha}=m 2^{1 / \alpha}
$$

then

$$
\mathcal{N}_{\mathcal{A}}(r, r / 2) \geq \mathcal{N}_{B}(r / 2)>m 2^{1 / \alpha}-m-1 .
$$

This is unbounded as $n \rightarrow \infty$; therefore, $\operatorname{dim}_{b}(\mathcal{A})=\infty$ by Lemma 2.4.

The Bouligand dimension of a geometric sequence is finite because geometric sequences have the scaling property needed for $\mathcal{N}_{\mathcal{A}}(r, r / 2)$ in Lemma 2.4 to be bounded. Moreover, we have:

Fact 4.2. Let $\mathcal{A}=\{0\} \cup\left\{a_{n} e_{n}: n \in \mathbf{N}\right\}$. If there exists $K$ and $\alpha$ such that $0<\alpha<1$ and

$$
(1 / K) \alpha^{n} \leq a_{n} \leq K \alpha^{n}
$$

then $\operatorname{dim}_{b}(\mathcal{A})=0$.

The proof of this fact follows from arguments similar to those used in the proof of Lemma 4.1. For the fractal dimension it is shown in [3] that $a_{n} \leq K / n^{\alpha} \operatorname{implies} \operatorname{dim}_{f}(\mathcal{A}) \leq 1 / \alpha$. To see that the lower bound in (4.1) is required consider

Theorem 4.3. There exist sequences converging arbitrarily fast to zero that have infinite Bouligand dimension. 
Proof. Let $\left\{b_{j}\right\}$ be a sequence converging to zero. Consider the sequence $\left\{a_{n} e_{n}\right\}$ where

$$
a_{n}=b_{j} \quad \text { for } n=2^{j-1}, \ldots, 2^{j}-1 .
$$

Let $B$ be the ball in $\mathcal{A}=\{0\} \cup\left\{a_{n} e_{n}: n \geq 1\right\}$ of radius $r=b_{j}+\epsilon$ centered at the origin. Thus

$$
B=\left\{a_{n} e_{n}: n \geq 2^{j-1}\right\} .
$$

Cover $B$ by $r / 2$ balls. Then

$$
\mathcal{N}_{\mathcal{A}}(r, r / 2) \geq \mathcal{N}_{B}(r / 2) \geq 2^{j}-2^{j-1}=2^{j-1}
$$

is unbounded; therefore, $\operatorname{dim}_{b}(\mathcal{A})=\infty$. Since no conditions on $\left\{b_{j}\right\}$ were imposed, then $\left\{a_{n}\right\}$ may converge arbitrarily fast to zero.

Corollary 4.4. There exist a set $\mathcal{A}$ such that $\operatorname{dim}_{b}(\mathcal{A})=0$ and $\operatorname{dim}_{b}(\mathcal{A}-$ $\mathcal{A})=\infty$.

Proof. Let $\left\{x_{j}\right\}$ be a sequence of the type given in Theorem 4.3 such that $\left\|x_{j}\right\| \leq 4^{-j}$. We assume the complement of the closed linear span of $\left\{x_{j}\right\}$ to be infinite dimensional and define $\left\{y_{j}\right\}$ to be an orthogonal sequence in that complement such that $\left\|y_{j}\right\|=4^{-j}$. Let $\mathcal{A}$ be the closure of the set $\left\{a_{j}\right\}$ where $a_{2 j}=y_{j}$ and $a_{2 j+1}=x_{j}+y_{j}$. Clearly the set of differences $\mathcal{A}-\mathcal{A}$ contains the set $\left\{x_{j}\right\}$ and therefore $\operatorname{dim}_{b}(\mathcal{A}-\mathcal{A})=\infty$.

Claim $\operatorname{dim}_{b}(\mathcal{A})=0$. If $k=2 j$ then

$$
\left\|a_{k}\right\|=\left\|y_{j}\right\|=4^{-j}=2^{-k}
$$

and so the condition (4.1) is satisfied with $\alpha=1 / 2$. If $k=2 j+1$, then

$$
\left\|a_{k}\right\| \leq\left\|x_{j}\right\|+\left\|y_{j}\right\| \leq 4^{-j}+4^{-j}=2\left(4^{-(k-1) / 2}\right) \leq 4\left(2^{-k}\right)
$$

and

$$
\left\|a_{k}\right\| \geq\left\|y_{j}\right\|=2\left(2^{-k}\right) .
$$

Therefore, taking $K=4$ and $\alpha=1 / 2$ satisfies (4.1) for all terms of the sequence $\left\{a_{j}\right\}$. It follows from Fact 4.2 that $\operatorname{dim}_{b}(\mathcal{A})=0$.

We will now use the same scaling properties exploited in the above examples to construct orthogonal projections that increase the Bouligand dimension. First, we need the following definitions and results.

Definition 4.5. Let $\vee \mathcal{A}$ denote the closed linear span of $\mathcal{A}$.

Lemma 4.6. There exists a projection $Q$ such that $Q \mathcal{A}$ contains an orthogonal sequence if and only if there exists $V \subseteq \mathcal{A}$ such that $\operatorname{dim}(\vee V)=\infty$ and

$$
\vee(V \backslash\{v\}) \neq \vee V \quad \text { for all } v \in V
$$


Proof. Suppose there is such a projection $Q$. Choose $v_{n} \in \mathcal{A}$ such that the $Q v_{n}$ form an orthogonal sequence. Define $V=\left\{v_{n}: n \in \mathbf{N}\right\}$. It follows that $Q v$ is orthogonal to $\vee(Q V \backslash\{Q v\})$ for any $v \in V$. Therefore $\vee(Q V \backslash\{Q v\}) \neq \vee(Q V)$. Since orthogonal projections are continuous and linear, then $Q$ commutes with $\vee$ and we obtain $Q(\vee(V \backslash\{v\})) \neq Q(\vee V)$, which implies $\vee(V \backslash\{v\}) \neq \vee V$.

Conversely, by omitting some elements of $V$ if necessary, we write $V=$ $\left\{v_{n}: n \in \mathbf{N}\right\}$ and suppose the orthogonal complement of $\vee V$ to be infinite dimensional. Let $f_{i}$ be an orthonormal sequence contained in that complement.

Define $V_{1}=V$ and $W_{1}=V_{1} \backslash\left\{v_{1}\right\}$. For induction suppose $\vee W_{n} \neq \vee V_{n}$. Then there exists $e_{n} \in \vee V_{n}$ such that $\left\|e_{n}\right\|=1$ and $e_{n} \perp \vee W_{n}$. Let $g_{n}=\left(e_{n}+f_{n}\right) / \sqrt{2}$. Define $V_{n+1}=V_{n} \cup\left\{g_{n}\right\}$ and $W_{n+1}=V_{n+1} \backslash\left\{v_{n+1}\right\}$. Clearly $\vee W_{n+1} \neq \vee V_{n+1}$.

The above construction yields an orthonormal sequence $g_{n}$ such that $\left(g_{i}, v_{i}\right) \neq 0$ and $\left(g_{i}, v_{j}\right)=0$ for $i \neq j$. Let $Q$ be the projection onto the space spanned by the $g_{n}$. It follows that $Q v_{n}=\alpha_{n} g_{n}$ where $\alpha_{n} \neq 0$ and so $Q V \subseteq Q \mathcal{A}$ contains an orthogonal sequence.

Theorem 4.7. If $\mathcal{A}$ satisfies Lemma 4.6, then there exists an orthogonal projection $P$ such that the Bouligand dimension of $P \mathcal{A}$ is infinite.

Note that Fact 4.2 provides an example of a set with finite Bouligand dimension that satisfies Lemma 4.6. However, a slight modification of the solution given by Halmos for Problem 11 in [12] yields an infinite dimensional compact set which does not satisfy these hypotheses. It is unknown whether the attractors of naturally occurring physical systems satisfy these hypothesis or not.

Proof of Theorem 4.7. By Lemma 4.6 there exists an orthogonal projection $Q$ such that $Q \mathcal{A}$ contains the orthogonal sequence $\alpha_{n} g_{n}$. Let

$$
m_{j}=\min \left\{\left|\alpha_{n}\right|: n=2^{j-1}, \ldots, 2^{j}-1\right\}
$$

and choose a monotone sequence $\beta_{j}$ strictly decreasing to zero such that $\beta_{j} \leq$ $m_{j}$. We may assume that the orthogonal complement of $\vee\left\{\alpha_{n} g_{n}: n \in \mathbf{N}\right\}$ in $Q H$ is infinite. Let $h_{n}$ be an orthonormal sequence in that complement and define

$$
\widetilde{g}_{n}=\sin \left(\theta_{n}\right) g_{n}+\cos \left(\theta_{n}\right) h_{n}
$$

where $\theta_{n}$ has been chosen in such a way that

$$
\alpha_{n} \sin \left(\theta_{n}\right)=\beta_{j} \quad \text { for } \quad n=2^{j-1}, \ldots, 2^{j}-1 .
$$

This can be done since $\beta_{j}<\alpha_{n}$. Let $P$ be the projection onto the space spanned by the $\widetilde{g}_{n}$. It follows that $P \mathcal{A}=P Q \mathcal{A}$ contains a sequence similar 
to the one found in Theorem 4.3 and therefore

$$
\operatorname{dim}_{b}(P \mathcal{A}) \geq \operatorname{dim}_{b}\left\{\alpha_{n} \sin \left(\theta_{n}\right) g_{n}: n \in \mathbf{N}\right\}=\infty .
$$

\section{The projection theorem.}

In this section we prove Theorem 1.2 restated more explicitly in the form of Theorem 5.2 below. We want to find rank $m$ orthogonal projections such that

$$
\|P x-P y\| \geq \epsilon f(\|x-y\|) \quad \text { for } x, y \in \mathcal{A}
$$

where $f$ is linear with a logarithmic correction. Moreover, we show that the measure of all the projections which do not satisfy this condition for any constant $\epsilon>0$ is zero. Writing $Y=\mathcal{A}-\mathcal{A}$ simplifies (5.1) to the requirement that

$$
\|P y\| \geq \epsilon f(\|y\|) \quad \text { for } y \in Y .
$$

Let $G$ be the space of orthogonal projections in $\mathbf{R}^{N}$ of rank $m$ and $\mu$ be the invariant measure on $G$ with respect to orthogonal transformations. Define the shadow of a set $B$ in $\mathbf{R}^{N}$ to be

$$
S(B)=\{P \in G: 0 \in P B\} .
$$

Showing that $\mu(S(Y))=0$ would prove almost every rank $m$ orthogonal projection is injective. To obtain continuity of the inverse we construct a slightly larger set $U$ containing $Y$ and show that $\mu(S(U))=0$. We shall use the following estimate in our computations:

Theorem 5.1. The measure of the shadow of a $\rho$-ball $B$ centered a distance $r$ from the origin is bounded by $\mu(S(B)) \leq C(\rho / r)^{m}$.

This result is given by Santalo in [22]. An explicit proof involving only elementary techniques has recently been given by by Friz and Robinson in $[11]$.

Theorem 5.2. Given a bounded set $\mathcal{A} \subseteq \mathbf{R}^{N}$ such that $\mathcal{A} \subseteq B_{R}(0)$ and $\operatorname{dim}_{b}(\mathcal{A}-\mathcal{A})<d<m$. Let $\eta>1$ and define

$$
f(x)=x\left(\frac{1}{\log _{2}(2 R / x)}\right)^{\eta /(m-d)}
$$

where $R=2 \sup \{\|a\|: a \in \mathcal{A}\}$. Then for almost every projection $P$ of rank $m$ there is a constant $\epsilon$ such that (5.1) is satisfied.

Proof. Define $r_{n}=R / 2^{n}$ and $\rho_{n}=f\left(r_{n-1}\right)$. Divide $Y$ into shells

$$
Z_{n}=\left\{y \in Y: r_{n} \leq\|y\| \leq r_{n-1}\right\}
$$

and cover $Z_{n}$ by balls of radius $\rho_{n}$. 
Let $Y=\mathcal{A}-\mathcal{A}$ and choose $K$ such that $\mathcal{N}_{\mathcal{B}}(r, \rho) \leq K(r / \rho)^{d}$ for all $\mathcal{B} \subseteq Y$. Further choose $c>2$ so large that $c^{m-d}>K$. Cover each $\rho_{n}$-ball by $\rho_{n} / c$ balls and each of those by $\rho_{n} / c^{2}$-balls and so on. Label the centers of the $\rho_{n} / c^{i}$-balls that cover $Z_{n}$ by $a_{n i j}$. Thus

$$
Z_{n} \subseteq \bigcup_{j} B_{\rho_{n} / c^{i}}\left(a_{n i j}\right)
$$

where the index $j$ ranges over at most $K^{i+1} c^{i d}\left(r_{n-1} / \rho_{n}\right)^{d}$ balls.

Since $c>2$, the covers formed by doubling the radius of the balls in each generation will be nested. Let

$$
U_{i}=\bigcup_{n, j} B_{2 \rho_{n} / c^{i}}\left(a_{n i j}\right)
$$

be the union of all the balls in the $i$-th generation with double the radius. For each $y \in Y$ there is a ball $B_{\rho}(a)$ of radius $\rho=\rho_{n} / c^{i}$ centered at $a$ such that $y \in B_{\rho}(a)$. If $P \notin S\left(U_{i}\right)$ then $0 \notin P B_{2 \rho}(a)$ and hence

$$
\|P y\| \geq \rho=\rho_{n} / c^{i}=f\left(r_{n-1}\right) / c^{i} \geq f(\|y\|) / c^{i}
$$

shows that (5.1) holds for the $i$-th generation with $\epsilon=1 / c^{i}$.

Let $U=\lim \sup _{i} U_{i}$. It remains to show the measure of $S(U)$ is zero. Estimate

$$
\begin{aligned}
\mu(S(U)) & \leq \mu\left(S\left(U_{i}\right)\right) \leq \sum_{n, j} \mu\left(S\left(B_{2 \rho_{n} / c^{i}}\left(a_{n i j}\right)\right)\right) \\
& \leq \sum_{n, j} C\left(\frac{2 \rho_{n}}{c^{i} r_{n}}\right)^{m} \leq \sum_{n} C K^{i+1} c^{i d}\left(\frac{r_{n-1}}{\rho_{n}}\right)^{d}\left(\frac{2 \rho_{n}}{c^{i} r_{n}}\right)^{m} \\
& =4^{m} C K\left(\frac{K}{c^{m-d}}\right)^{i} \sum_{n}\left(\frac{f\left(r_{n-1}\right)}{r_{n-1}}\right)^{m-d} \\
& =4^{m} C K\left(\frac{K}{c^{m-d}}\right)^{i} \sum_{n} \frac{1}{n^{\eta}}=L\left(\frac{K}{c^{m-d}}\right)^{i}
\end{aligned}
$$

where $L$ is a constant. Letting $i$ become large, we obtain $\mu(S(U))=0$. Thus, almost every orthogonal projection of rank $m$ satisfies (5.1) for some $\epsilon>0$.

Acknowledgments. I am grateful towards Professor Ciprian Foias for reading over earlier versions of this paper and providing helpful comments and discussions. I also enjoyed many useful discussions with Professor James Robinson. This work was supported in part by the National Science Foundation under grant number DMS-9706903. 


\section{References}

[1] P. Assouad, Étude d'une dimension métrique liée à la possibilité de plongements dans $\mathbf{R}^{n}$, C.R. Acad. Sci., Paris, Sér. A-B, 288(15) (1979), A731-A734, MR 80f:54030, Zbl 0409.54020.

[2] _ Plongements Lipshitziens dans $\mathbf{R}^{n}$, Bull. Soc. Math. France, 111 (1983), 429-448, MR 86f:54050, Zbl 0597.54015.

[3] A. Ben-Artzi, A. Eden, C. Foias and B. Nicolaenko, Hölder continuity for the inverse of Mañé's projection, Journal of Mathematical Analysis and Applications, 178(1) (Sept. 1, 1993), 22-29, MR 94d:58091, Zbl 0815.46016.

[4] Georges Bouligand, Ensembles impropres et nombre dimensionnel, Bull. Sci. Math., 52 (1928), 320-344, 361-376.

[5] P. Constantin, A construction of inertial manifolds, in 'The Connection between Infinite Dimensional and Finite Dimensional Dynamical Systems', Contemporary Mathematics, 99 (1987), 27-62, MR 91a:58040, Zbl 0691.58040.

[6] P. Constantin, C. Foias, B. Nicolaenko and R. Temam, Integral and Inertial Manifolds for Dissipative Partial Differential Equations, Springer-Verlag, Applied Math. Sciences, 70, New York, 1989, MR 90a:35026, Zbl 0683.58002.

[7] _ Spectral barriers and inertial manifolds for dissipative partial differential equations, J. Dynamics and Differential Equations, 1 (1989), 45-73, MR 90i:35234, Zbl 0701.35024

[8] A. Eden, C. Foias, B. Nicolaenko and R. Temam, Exponential Attractors for Dissipative Evolution Equations, John Wiley and Sons, 1995, MR 96i:34148, Zbl 0842.58056.

[9] K. Falconer, Fractal Geometry: Mathematical Foundations and Applications, John Wiley and Sons, 1990, MR 92j:28008, Zbl 0689.28003.

[10] C. Foias and E. Olson, Finite fractal dimension and Hölder-Lipschitz parameterization, Indiana University Mathematics Journal, 45(3) (Fall 1996), 603-616, MR 97m:58120, Zbl 0887.54034.

[11] P.K. Friz and J.C. Robinson, Constructing an elementary measure on a space of projections, preprint.

[12] P.R. Halmos, A Hilbert Space Problem Book, 2nd ed., Springer-Verlag, 1982, MR 84e:47001, Zbl 0496.47001.

[13] B.R. Hunt and V.Yu. Kaloshin, Regularity of embeddings of infinite-dimensional fractal sets into finite-dimensional spaces, Nonlinearity, 12(5) (1999), 1263-1275, MR 2001a:28009, Zbl 0932.28006.

[14] B.R. Hunt, T. Sauer and J.A. Yorke, Prevalence: A translation-invariant almost every for infinite dimensional spaces, Bull. Amer. Math. Soc., 27 (1992), 217-238, MR 93k:28018, Zbl 0763.28009; Prevalence: An addendum, Bull. Amer. Math. Soc., 28 (1993), 306-307, MR 93k:28019, Zbl 0782.28007.

[15] J. Mallet-Paret and G. Sell, Inertial manifolds for reaction diffusion equations in higher space dimensions, J. Differential Equations, 22 (1988), 805-866, MR 90h:58056, Zbl 0674.35049.

[16] R. Mañé, On the dimension of the compact invariant sets of certain non-linear maps, Lecture Notes in Mathematics, 898, Springer-Verlag, (1981), 230-242, Zbl 0544.58014. 
[17] Ladislav Mišík Jr. and Tibor Žáčik, On the metric dimension of converging sequences, Comment. Math. Univ. Carolinae, 34(2) (1993), 367-373, MR 95d:40020, Zbl 0845.54026.

[18] H. Movahedi-Lankarani, On the inverse of Mañés projection, Proceedings of the American Mathematical Society, 116(2) (Oct. 1992), 555-560, Zbl 0777.54011.

[19] B. Nicolaenko and W. Qian, Inertial manifolds for nonlinear viscoelasticity equations, Nonlinearity, 11(4) (1998), 1075-1093, MR 99d:35164, Zbl 0914.35020.

[20] J.C. Robinson, Global attractors: Topology and finite-dimensional dynamics, Journal of Dynamics and Differential Equations, 11(3) (1999), 557-581, MR 2000k:37123, Zbl 0953.34049.

[21] _ All possible chaotic dynamics can be approximated in three dimensions, Nonlinearity, 11 (1998), 529-545, MR 99d:58124, Zbl 0918.34048.

[22] L.A. Santalo, Integral Geometry and Geometric Probability, Addison-Wesley Publishing Company, 1976, MR 55 \#6340, Zbl 0342.53049.

[23] T. Sauer, J.A. Yorke and M. Casdagli, Embedology, J. Stat. Phys., 71 (1993), 529-547, MR 93c:58147, Zbl 0943.37506.

Received April 5, 2000 and revised November 3, 2000.

Department of Mathematics

103 Multipurpose Science \& Technology Bldg.

University of CALifornia, Irvine

IRVINE, CA 92697-3875

E-mail address: ejolson@fractal.math.unr.edu 